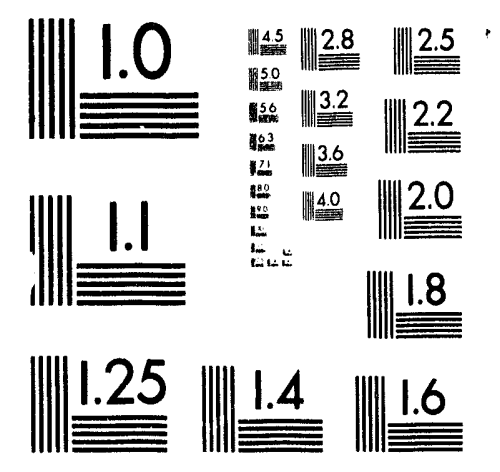



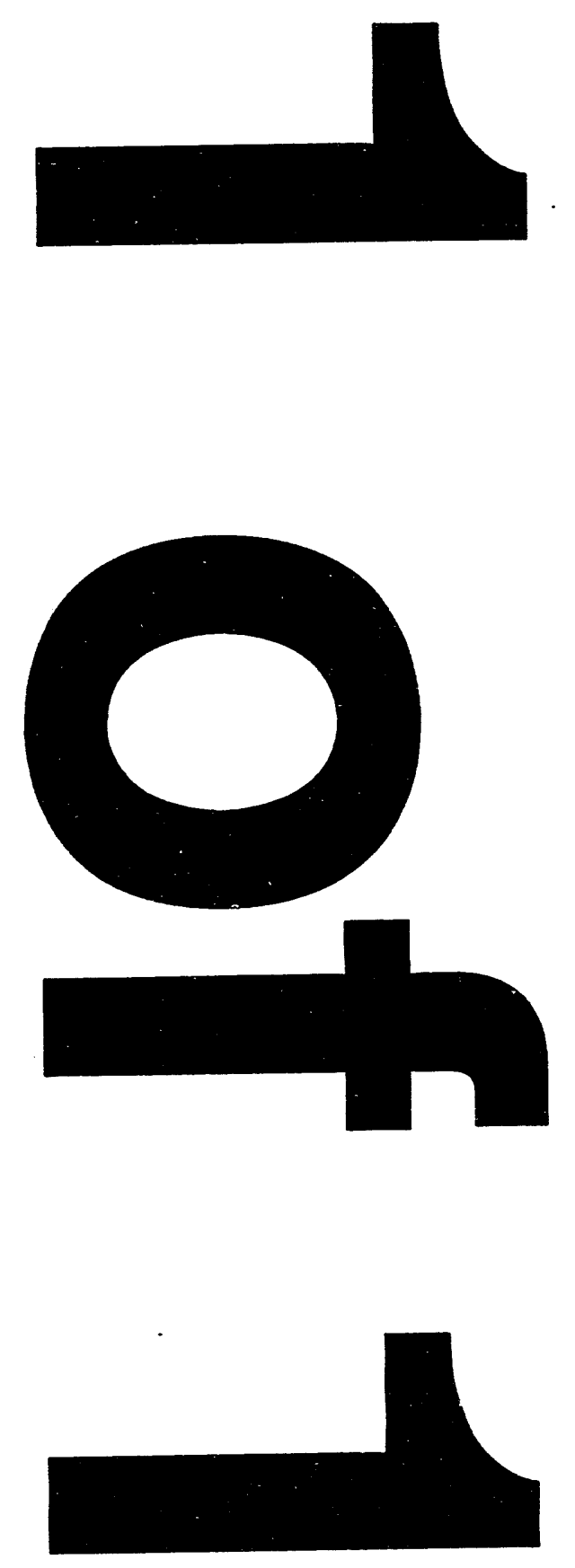


\title{
The Deposition of Boron Nitride and Carbon Films on Silica Glass Fibers
}

by

W. L. Smith, T. A. Michalske, \& R. R. Rye

Surface \& Interface Sciences

Sandia National Laboratories

Albuquerque, New Mexico 87185-0344

\begin{abstract}
A chemical vapor deposition technique is used to produce amorphous boron nitride and carbon thin films on high strength silica glass fibers. In this method, the fiber is drawn under ultra high vacuum conditions and low pressure process gases, in the presence of a hot tungsten filament, are used to grow films at low substrate temperatures. Films deposited with this technique do not degrade the intrinsic pristine strength of the silica fibers under dry conditions and, when stressed in chemically aggressive environments, act as effective barrier coatings.
\end{abstract}




\section{Introduction}

Silica based optical fibers are rapidly assuming a pivotal role in advanced energy and defense related technologies. The reliable performance of these fibers is sensitive to factors that effect the long term mechanical strength and time dependent degradation of the optical transmission. It has been shown ${ }^{(1)}$ that exposure to water can reduce the strength of glass fibers with time under stress. It is also known that exposure of the optical fibers to hydrogen gas can degrade the optical transmission of optical fiber cables. Because of these effects, it is extremely important to protect the intrinsic strength properties of the fiber during formation and to maintain the extrinsic fiber strength by isolating the fiber from the contact or contamination after formation.

An important means of protecting the pristine properties of silica glass optical fibers involves the application of barrier coatings. To date, coatings made from boron nitride (BN) and carbon (C) and other materials have been applied to fibers. Although many of these coatings have been shown to provide adequate protective barriers, in every case the coating process itself results in a substantial decrease in the measured mechenical strength when compared to pristine glass fibers ${ }^{(1)}$. The coating process described in this report is a method by which the desired coating can be applied with little or no degradation of the pristine strength of the glass fiber.

\section{Fiber Formation}

In order to form contamination-free fiber surfaces, we use an ultra high vacuum (UHV) chamber as a means of excluding particulate and chemical species during the fiber fabrication process. Due to the high softening point $\left(>1700^{\circ} \mathrm{C}\right.$ ) of fused quartz, an intense heat source is required to draw fibers. Since a carbon dioxide laser produces radiation at a nominal wavelength of $10.6 \mu \mathrm{m}$ and the optical transmission of fused quartz is essentially zero above $4.0 \mu \mathrm{m}$, this laser light provides an excellent heat source for this application. Fiber sections on the order of $10 \mathrm{~mm}$ long and $30 \mu \mathrm{m}$ in diameter are drawn from a $1.0 \mathrm{~mm}$ preform section of silica glass rod. Critical fiber formation parameters are monitored and controlled through the use of a microprocessor and digital image processor. After formation, the resulting fiber sections can be coated and tested to failure under controlled environmental conditions. Since fiber drawing, coating, and testing are conducted in situ, the handling, fixturing, and contamination problems associated with delicate fiber sections are eliminated. This system has been previously described in detail ${ }^{(2)}$.

\section{Fiber Strength}

As part of any effort to understand the performance of coated fibers, it is first necessary to examine the strength of uncoated pristine fibers that have not been exposed to abrasive or chemically reactive agents. Studies of high strength silica glass fibers in the pristine condition allow us to assess the potential of optical fibers and provide a basis for the examination, in a controlled fashion, of the role of chemical species in degrading the fiber strength as well as the possible beneficial effects of barrier coatings.

The intrinsic strength of a silica glass fiber is a measure of the perfection of the glass surface and the glass structure. Since the flaws which control this strength are too small for observation, the fracture strength can be used as an estimate of the distribution of these flaws. The extrinsic strength of silica glass fibers is controlled by macroscopic flaws such as abrasions and oxide contaminants which influence strength behavior. This reduction in strength is observed as a slope transition from intrinsic values on a Weibull distribution plot.

A moderating factor which comes to play in strength behavior is the environment in which the glass fiber is fractured. Fibers are commonly formed and/or tested in room temperature laboratory air which contains a substantial percentage of water. The newly formed fibers have a pristine strength as high as $14 \mathrm{GPa}$. As the fiber cools from the drawing process, the silica glass surface is hydrated by water in the air. When the fiber is eventually tested in air, the stress corrosion effects ${ }^{(3)}$ of adsorbed surface water results in a distribution of strengths in the range of $3 \mathrm{GPa}$ to $6 \mathrm{GPa}^{(4,5)}$ which is well below the average strengths $(>10 \mathrm{GPa})$ for pristine fibers when tested under UHV conditions. While it is clear that the fatigue behavior of low strength fibers is controlled by extrinsic flaws and reactive chemical species, little information is available which relates to the behavior of much higher strength pristine fibers. By 
producing and testing fibers relatively free of the effects of external strength degradation, we are able to investigate a higher range of strength values and are able to more clearly evaluate the effects of coatings and coating processes.

\section{Coating Process}

A modified chemical vapor deposition (CVD) process $^{(6)}$ is used to apply coatings of boron nitride and carbon to freshly drawn silica glass fibers. A major source of strength reduction, which results from a reaction between the hot silica surface and the coating material in standard CVD processes, is reduced with the use of a low temperature, filament activated CVD process. In this method, a hot tungsten filament $\left(\approx 1400^{\circ} \mathrm{C}\right.$ for boron nitride and $\approx 2200^{\circ} \mathrm{C}$ for carbon) is placed in proximity with the silica fiber during the deposition process. A precursor gas of borazine $\left(\mathrm{B}_{3} \mathrm{~N}_{3} \mathrm{H}_{6}\right)$ is used for the production of $\mathrm{BN}$ films while cyclopentane $\left(\mathrm{C}_{5} \mathrm{H}_{10}\right)$ gas is used for the production of $\mathrm{C}$ films. In this process the thermal activation of the precursor gas occurs at the surface of the hot filament, not at the pristine glass surface. Since only the filament is heated, wall reactions are eliminated or greatly reduced and fiber temperature is limited to the radiative heating from the adjacent hot filament. Maximum filament temperatures and the corresponding maximum depcsition rates are limited by high temperature reactions between the precursor gases and the tungsten filament which occur above $2200^{\circ} \mathrm{C}$. Since the coatings are applied immediately after fiber formation, at low temperatures, and under controlled environmental conditions, the pristine strength of the glass fiber is preserved.

Films on the order of $25,000 \AA$ have heen produced using low temperature, filament activated CVD. The low temperature ( $<250^{\circ} \mathrm{C}$ substrate) BN films show no indication of crystallinity with $x$-ray diffraction (XRD). This reduced crystallinity, a natural consequence of low temperature film formation, can be an important conisideration for coating application. As the substrate deposition temperature is increased, the crystallinity increases proportionally. Clearly, this difference in crystallinity, which is directly related to substrate deposition temperature, explains the tendency for BN films deposited at very low substrate temperatures to slowly degrade when exposed to air. BN films deposited at somewhat higher $\left(>400^{\circ} \mathrm{C}\right.$ ) temperatures show a marked resistance to this decomposition. X-ray photoelectron spectra (XPS) show the films to have a B:N ratio (within our experimental error) of $1: 1$ with no other detectable impurities above the $0.5 \%$ level. Both Raman and infrared (IR) spectroscopy are characteristic of h-BN with small amounts of hydrogen detected as N-H and B-H bands in the IR spectrum. The carbon films produced with this technique also exhibit XPS impurity levels below $0.5 \%$ and no XRD evidence of crystallinity is observed. Raman spectra show no long range order. The carbon films are stable in air regardless of substrate deposition temperature.

\section{Discussion}

Much of the initial work in this study has been directed toward the development of the low temperature, filament activated CVD process for the deposition of $\mathrm{C}$ and BN films. The deposition of carbon films on fibers shows promise and recent developments indicate that well consolidated, glassy films can be deposited in a temperature range between $250^{\circ}$ and $350^{\circ} \mathrm{C}$. These films do not degrade with time when exposed to the atmosphere and serve as effective barrier coatings.

BN films can be formed at very high rates but, when deposited at very low temperatures, have proven to be less effective as barrier materials. While low temperature deposition is desirable, it is not clear, in the case of silica fibers, how low the substrate temperature must be kept to continue to preserve the intrinsic strength of the fiber. It may be possible to increase the deposition temperature, with a corresponding increase in crystallinity, to an acceptable level, and still maintain the desired strength characteristics. The development of these coatings, with the proper characteristics, offer many advantages over current deposition techniques.

Recently, one of our studies has resulted in the suciessful deposition of $\mathrm{C}$ coatings on low melting temperature, infra-red transmitting glass fibers used in medical laser applications. Since fibers made from this type of glass are extremely hygroscopic, the successful development of an adequate hermetic coating is very desirable. 


\section{Summary}

We have developed a unique process for the formation, deposition, and testing of high strength silica fibers. Silica fibers with maximum inert strengths of 14 $\mathrm{GPa}$ are produced under controlled environmental conditions. Boron nitride and carbon barrier films can be deposited on fibers at temperatures below $250^{\circ} \mathrm{C}$ using a low temperature, filament activated CVD process. An primary feature of this process is the separation between the thermal activation step and the surface reaction step in film deposition. Films deposited in this manner do not degrade the average intrinsic strength of the silica glass fibers.

\section{References}

1. C. R. Kurkjian, J. T. Krause, \& M. J. Matthewson, "Strength and Fatigue of Silica Optical Fibers", J. Lightwave Tech. Vol. 7, [9] (1989), 7.
2. W. L. Smith, "Automated Glass Fiber Drawing", Sandia National Laboratories, SAND89-1674, (1989).

3. T. A. Michalske \& B. C. Bunker, "Slow Fracture Model Based on Strained Silicate Structures", J. Appl. Phys. 56, [10], (1984).

4. U. C. Paek, C. D. Spainhour, C. M. Schroeder, \& C. R. Kurkjian, "Tensile Strength of 50m-Long Silica Fibers Drawn with a Galvanometer Scanning System", J. Am. Ceram. Soc., Vol. 59, [6], 630-633, (1980)

5. W. J. Duncan, P. W. France, \& S. P. Craig, "The Effect of Environment on the Strength of Optical Fiber", in Strength of Inorganic Glass, Edited by C. R. Kurkjian, Plenum Press, NY, (1985).

6. R. R. Rye, "Hot Filament Activated Chemical Vapor Deposition of Boron Nitride”, J. Vac. Sci. Technol. A 9 (3), May/Jun, (1991). 


\section{DISTRIBUTION:}

$\begin{array}{llrl}\text { MS 0161 } & 11510 & 3 & \text { Intellectual Property Development } \\ \text { MS 0344 } & 1114 & 1 & \text { T. A. Michalske } \\ \text { MS 0344 } & 1114 & 1 & \text { R. R. Rye } \\ \text { MS 0344 } & 1114 & 20 & \text { W. L. Smith } \\ \text { MS 0619 } & 7151 & 1 & \text { Technical Publications } \\ \text { MS 0899 } & 7141 & 5 & \text { Technical Library } \\ \text { MS 1119 } & 7613-2 & 10 & \text { Document Processing } \\ \text { MS 9018 } & 8523-2 & 1 & \text { Central Technical Files }\end{array}$

\section{Patent Interest}

The U.S. Patent and Trademark Office has issued patent $\# 5,246,746$ to T. A. Michalske, R. R. Rye, and W. L. Smith for the invention of Hermetic Coatings for Optical Fibers by Hot Filament Assisted Chemical Vapor Deposition. 

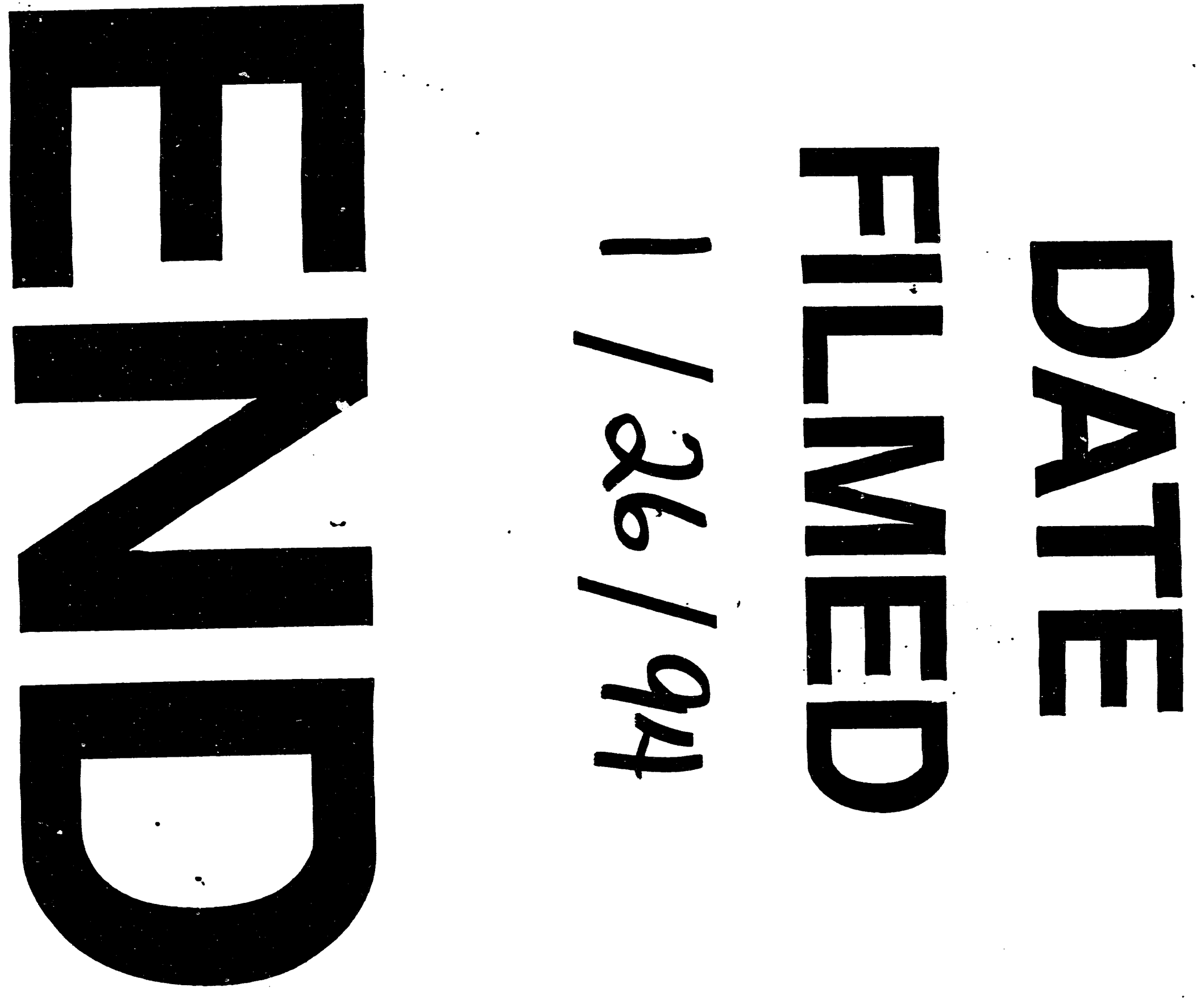
